\title{
OSA Patient Monitoring System Based on the Internet of Things Framework
}

\author{
Cai Liangming \\ C\& IE College Fuzhou, China \\ University of South Alabama \\ Zhicheng College \\ m161110009@fzu.edu.cn \\ Fuzhou University
}

\author{
Le Jingrong \\ Zhicheng College \\ Fuzhou University \\ jingrongle@fzu.edu.cn \\ Ruan Xuxin \\ Zhicheng College \\ Fuzhou University \\ xuxinruan@fzu.edu.cn
}

\author{
$\mathrm{Du}$ Min * \\ Provincial Key Laboratory of Eco- \\ industrial Green Technology \\ Wuyi College \\ Wuyishan, China \\ Fuzhou University \\ dumin@fzu.edu.cn
}

\begin{abstract}
Sleep respiratory diseases include obstructive sleep apnea (OSA) and central nervous system Sleep apnea (CSA) and so on, the research shows that $4 / 5$ of the patients with sleep apnea syndrome have snoring phenomenon. Long term sleep apnea can cause system dysfunction and multiple diseases, such as increasing the risk of obesity and heart disease, and even sudden death. According to the World Health Group, the high-risk groups of sleep respiratory diseases mainly involve obese patients and the elderly. In the middle-aged men and women in the United States, OSA incidence the number of sleep disordered breathing patients in the world is a large group, and nearly $80-90 \%$ of them have apnea syndrome. but they undiagnosed. Therefore, the monitoring and auxiliary regulation of sleep disordered breathing are of great significance for the prevention and diagnosis of sleep and related diseases. The way is to help patients get normal Body Mass Index (BMI), but the patients cannot return to normal because their perseverance is insufficiency, so the method can not achieve the goal of reducing sleep disorder (SD). This paper presents an OSA patient interactive monitoring systembased on the Internet of Things (IoT) framework. This system allows OSA patients to get timely rescue when they are sleepy outside. Because the Beidou position marker has an interactive function, it can reduce the anxiety of the patient while waiting for the rescue. At the same time, if a friend helps the OSA patients to call the doctor, the friend can also report the patient's condition in time. This system uses the popular IoT framework. At the bottom is the data acquisition layer, which uses wearable sensors to collect vital signs from patients, with a focus on ECG and $\mathrm{SpO} 2$ signals. The middle layer is the network layer that transmits the collected physiological signals to the Beidou indicator using the Bluetooth Low Energy (BLE) protocol. The top layer is the application layer, and the application layer uses the
\end{abstract}

mature rescue interactive platform of Beidou. Since the GPS indicator has not included the communication satellite, So it has no SMS function. OSA patients can only passively wait for a rescue. Moreover, due to the lack of satellites in Asia and the insufficient density of the ground-enhanced system, the positioning error of OSA patients is large. The Beidou system developed by China itself, the main coverage of the satellite is in Asia, and is equipped with a high-density ground-based augmentation system. Therefore, the Beidou model improves the positioning accuracy and is equipped with a special communication satellite, which increases the short message interaction function. Therefore, patients can report disease progression in time while waiting for a rescue. After our simulation test, the effectiveness of the OSA patient rescue monitoring system based on the Internet of Things framework and the positioning accuracy of OSA patients have been greatly improved. Especially when OSA patients work outdoors, the cell phone base station signal coverage is relatively weak. The satellite signal is well covered, plus the SMS function of the Beidou indicator. Therefore, the system can be used to provide timely patient progress and provide data support for the medical rescue team to provide a more accurate rescue plan. After a comparative trial, the rescue rate of OSA patients using the detection device of this system was increased by 15 percentage points compared with the rescue rate using only GPS satellite phones.

Keywords: IoT, OSA patient rescue system, Beidou indicator.

\section{INTRODUCTION}

Sleep respiratory diseases include obstructive sleep apnea (OSA) and central nervous systemSleep apnea (CSA) and so on, the research shows that 4 / 5 of the patients with sleep apnea syndrome have snoring phenomenon [1]. Long term sleep apnea can cause system dysfunction and multiple 
diseases, such as increasing the risk of obesity and heart disease, and even sudden death [2]. According to the World Health Group, the high-risk groups of sleep respiratory diseases mainly involve obese patients and the elderly [3]. In the middle-aged men and women in the United States, OSA incidence the number of sleep disordered breathing patients in the world is a large group, and nearly $80-90 \%$ of them have apnea syndrome. but they undiagnosed[4]. Therefore, the monitoring and auxiliary regulation of sleep disordered breathing are of great significance for the prevention and diagnosis of sleep and related diseases. The way is to help patients get normal Body Mass Index (BMI), but the patients cannot return to normal because their perseverance is insufficiency, so the method can not achieve the goal of reducing sleep disorder $(\mathrm{SD})$ [5].

There are approximately 5,000 standard sleep laboratonies in the world, and most sleep laboratories use polysomnography (PSG) as the gold standard for detecting sleep dis orders [6]. PSG can only be used in specialized clinics or hospitals under constant medical supervision to collect physiological signals from patients with sleep disorders, and then use professional sleep experts to diagnose patients who are sleep dis orders based on physiological signals detected by PSG [7] This means that patients must go to specialized medical institutions, which will inevitably increase the burden on patients. Moreover, PSG requires the integration of many sensors on the human body, which is considered invasive, so PSG screening can interfere with sleep [8]. In addition, PSG is costly and time consuming, plus the need for professional sleep experts. Therefore, PSG is difficult to become a method for long-term monitoring of patients with sleep disorders [9]. Limited data show that patients with severe sleep apnea who do not receive effective treatment will increase the risk of sudden cardiac death. If there is no heart disease, only sleep apnea, the possibility of death is very small. Long-term sleep apnea, if not well treated, will increase the incidence of a series of cardiovascular diseases including hypertension, pulmonary hypertension, coronary heart disease, heart failure, etc., and will also make the therapeutic effect of these diseases worse. For example, about $70 \%$ of refractory hypertension patients with poor blood pressure control have sleep apnea. Therefore, screening sleep apnea is very important for hypertension patients whose blood pressure is difficult to control. Sleep apnea may also increase the incidence of diabetes, fatty liver, stroke and depression. It can make people sleepy and lack of attention during the day, which also increases the possibility of accidents such as traffic accidents or the operation of other dangerous equipment.

The data suggest that $80 \%$ of patients with sleep disorders are not effectively diagnosed, even if someone is diagnosed with obstructive sleep disorder (OSA), they are not treated in time. The reason is that, on the one hand, surgery can not effectively solve the problem of OSA. On the other hand, sleep correction using CPAP can cause discomfort to patients with sleep disorders. There is therefore a need for an Internet of Things (IoT) framework system to monitor OSA patients.
This systemenables OSA patients to be rescued in time when they are drowsy outdoors. Because the Beidou position marker has an interactive function, it can reduce the anxiety of the patient while waiting for rescue. At the same time, if a friend helps an OSA patient to call a doctor, the friend can also report the patient's condition in time. The system uses a popular IoT framework. At the bottom is the data acquisition layer, which uses wearable sensors to collect vital signs from patients, with a focus on ECG and $\mathrm{SpO} 2$ signals. The middle layer transmits the collected physiological signals to the network layer of the Beidou indicator using the Bluetooth Low Energy (BLE) protocol. The top layer is the application layer, and the application layer uses the mature Beidou rescue interactive platform. Since the GPS indicator does not include a communication satellite, it does not have an SMS function. OSA patients can only passively wait for rescue. In addition, due to the lack of satellites in Asia and the insufficient density of ground-enhanced systems, OSA patients have large positioning errors. The Beidou system developed by China itself, the main coverage of the satellite is in Asia, and is equipped with a high-density ground enhancement system Therefore, the Beidou model improves the positioning accuracy and is equipped with a special communication satellite, which increases the short message interaction function. Therefore, patients can report disease progression in a timely manner while waiting for a rescue. After our simulation test, the effectiveness of the OSA patient rescue monitoring systembased on the Internet of Things framework and the positioning accuracy of OSA patients have been greatly improved. Especially when OSA patients work outdoors, the signal coverage of cell phone base stations is relatively weak. The satellite signal is well covered, plus the SMS function of the Beidou indicator. As a result, the system can be used to provide timely patient progression and provide data support to the medical rescue team to provide a more accurate rescue plan. After a comparative test, the rescue rate of OSA patients using the system's detection equipment increased by 15 percentage points compared to the rescue rate using only GPS satellite phones.

The rest of this paper is structured as follows. Part 2 presents the latest literature on this area of research. Part 3 presents the methods and simulation results of a sleep dis order monitoring system based on the Internet of Things (IoT) framework. Section 4 summarizes and suggests the direction for future improvements.

\section{RELATED WORK}

At present, the golden standard for the diagnosis of sleep apnea is polysomnography (PSG) [10]. However, PSG is expensive, so it is necessary for special sleep respiratory monitors to connect electroencephalogram (EEG), eye movement, EMG and other wires to the body surface of patients, so that their application is limited to a certain extent [11]. In order to reduce the measured physiological signals, the detection method of sleep dyspnea based on SpO2, ECG 
(Electrocardiogram) signal has become a hot research topic [12].

In the research of detecting sleep dyspnea based on ECG signal, the methods of OSA detection of ECG signal were studied by the University of Wisconsin, Taiwan Jiaotong University and Peking University [11] --[15]. At present, there are severalCFDA certified devices in China to diagnose sleep dyspnea by sticking electrodes in the human body [16]. The above studies show the value of respiratory and heart rate variability in diagnosis and sleep staging, but the detection of ECG may be affected by the location of the electrode, poor contact or electrode shedding caused by action. Photoelectric volume pulse wave photolethysmograph (PPG) signal morphology is like arterial blood pressure waveform, which can realize the extraction of a variety of physiological parameter information, which makes PPG become a popular non-invasive physiological parameter information monitoring tool in the scientific community [17]. Although the academic research of portable sleep monitor using PPG signal has begun to increase, and there are some commercial products such as smart bracelet on the market, such as Finland Beddit, Israel Earlysense and French Withings [16] --[21]. But such products are usually identified and roughly analyzed for deep or shallow sleep based on heart rate and body movement data. This model only serves as a reference. cannot completely replace the polysomnography [22].

Beidou satellite navigation system provides positioning, navigation and timing service [23], which is divided into open service [24] and authorized service [25]. Licensing services: in addition to free and open services to the world, there are services that require an authorization, which is divided into different levels and is distinguished between military and civilian use [26]. Beidou satellite navigation system is very important to call for help and position when in distress at sea. In view of this situation, IMO has developed a set of global maritime distress and safety system (GMDSS) [27]. The system consists of three major parts: INMARSAT (Inmarsat system) [28] and COS-PAS-SARSAT (Polar-orbiting Satellite Search and Rescue System) [29], ground-based radio communication system(i.e. Coast Station) [30] and Maritime Safety Information dis semination system[31].

The above studies show the feasibility of using machine learning to study the auxiliary OSA system [32]. However, most of the above studies are based on small sample data sets and lack large-scale clinical applications. Moreover, it is possible to select too many features in feature engineering, resulting in too much computation[33]. Or choose too few features, resulting in low classification accuracy. In addition, when selecting the deep learning clas sification method, setting the parameters manually is too inefficient. At the same time, it is possible to over-train and cause the test to be over-fitting. At the same time, since most research methods are only based on small data sets, the generalization ability of the model is low when the actual application is in hospital. Therefore, this topic intends to use machine learning algorithms on the cloud platform to normalize blood oxygen saturation and ECG signals from different devices.

\section{METHOD AND SIMULATION RESULTS}

The hardware component of the IOT-based sleep disorder monitoring systemconsists of three parts. The bottomlayer is the hardware terminal of the indicator mark, the middle layer is the transmission medium, and the upper layer is the application platform. The beacon terminal includes an ARM main control board, a 4th generation mobile communication module, a Beidou communication module, a Bluetooth communication module, an antenna interface module, and a power management module. In addition, the sides of the housing are provided. There are data transmission interfaces, SOS buttons and charging ports. There are several sealing rings and fixing screws at the bottomof the housing. However, when a sleep disorder patient is working outdoors, when the drowsiness state occurs, the SOS button can be used to send the help information to the Beidou satellite. Through the auxiliary ground-based augmentation system, Beidou Satellite sends the current status and position coordinates of the sleepdisabled patients to the Beidou rescue platform. The information released by the rescue platform enables the rescue team to quickly and accurately find the location to be rescued. As shown in Figure 1.

Logistic regression is a statistical tool based technique to deal with machine learning problems. It is generally applied for binary classification. Logistic regression works with sigmoid function. As shown in Fig 4, the accuracy of the LR model is very high (90\%). In particular, LR models using all 10 features show better accuracy than other existing models using RQA functionality with an accuracy rate of $90 \%$.

Most importantly, models using LR are still the best in terms of prediction accuracy, precision, recall, and F-measure.

The results of scatter plot from logis tic regression is shown in Fig. 1. It is very clear from the figure that logistic regression has perform efficiently to solve sleep apnea. The ROC curve and parallel co-ordinate plot is shown in Fig. 2 and Fig. 3. Parallel co-ordinate plot also shows that the prediction is accurate. From confusion matrix in Fig. 4, it can be seen that the accuracy is $90 \%$. Moreover, the figure also shows the true positive rate and false negative rate. The confusion matrix with true class and predicted class is shown in Fig. 5. The Fig. 5 shows that positive predictive value is $90 \%$ and false discovery rate is $10 \%$. Fig. 6 consists of true class and predicted class. 


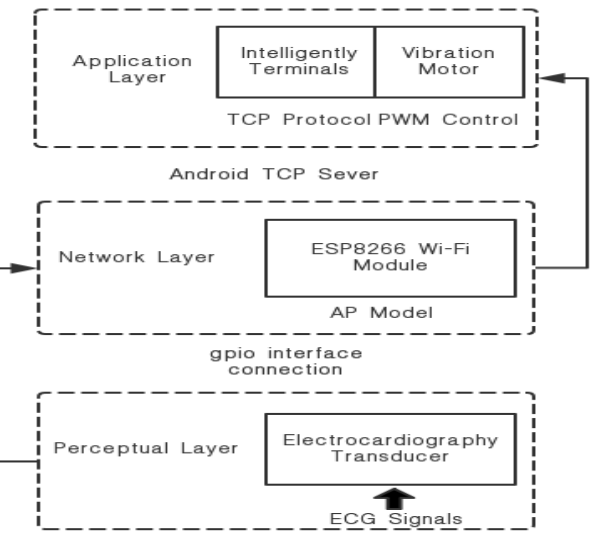

Fig. 1 OSA patient monitoring systemstructure

\section{A. Design of the Perceptual Layer}

\subsection{Hardware design}

The whole system is based on ARM main control board, 4th generation mobile communication module, Beidou communication module, Bluetooth communication module, antenna interface module and power management module. In addition, the sides of the housing are provided. There are data transmission interfaces, SOS buttons and charging ports. There are several sealing rings and fixing screws at the bottom of the housing. However, when a sleep disorder patient is working outdoors, when the drowsiness state occurs, the SOS button can be used to send the help information to the Beidou satellite. The Beidou second-generation passive positioning and Beidou active short message communication are combined. The location information of OSA patients can be obtained through the Beidou module, while OSA patients can send disease information through Android mobile phone software operation. The emergency call function is provided in an emergency, and the OSA patient can send his own location information and simple condition information, thereby realizing the emergency rescue function of the OSA patient monitoring system based on the IOT framework.

\section{1.1 Function design of obtaining position information}

The STM32 development board communicates with the Beidou module by serial port. It uses the command of RNSS format and uses BDGGA to get its own location information and stores it in the cache. When a mobile phone is connected with the terminal through Bluetooth, the data is forwarded to the mobile phone if the mobile phone makes a data request. The mobile phone is transformed into latitude and longitude coordinates through the received Beidou position information and displayed.

B. Design of the Network Layer

3. 1.2 functional design of the mobile phone
OSA patients can use the Android phone to send AT commands to initialize the Beidou indicator, and obtain their own location information through BLE communication. The latest rescue information and the latest location information obtained through the refresh function. The OSA can also provide a special message input function through the Beidou indicator, input the condition information through the mobile phone and forward it to the terminal, and then the condition message and location information can be sent to the Beidou rescue platform by the Beidou indicator.

3. 1.3 Design of communication protocolbetween end and terminal of mobile phone

It is very necessary for the mobile terminal to communicate with the terminal using Bluetooth to maintain complete communication. The mobile phone terminal obtains whether the communication is normal by sending instructions, and the terminal receives the correct return value by continuously sending test information. And provide led flicker function display.

\section{1.4 Terminal automatic help function}

By setting the timer, the function of obtaining the position can be used automatically, and the acquired position information can be sent periodically. Start the B4 function by triggering the button B3 automatic function to stop the automatic function.

The main consideration is to obtain location information, and how to send information for help and other aspects. In the event of a problem, the rescue party can get the message in time, and the suitor can also get the necessary information anytime and anywhere. The interactive function of the display object is mainly realized by Bluetooth module and STM32 main control board.

\subsection{SOFTWARE DESIGN}

3.2.1 Terminal part system design: as shown in figure 2

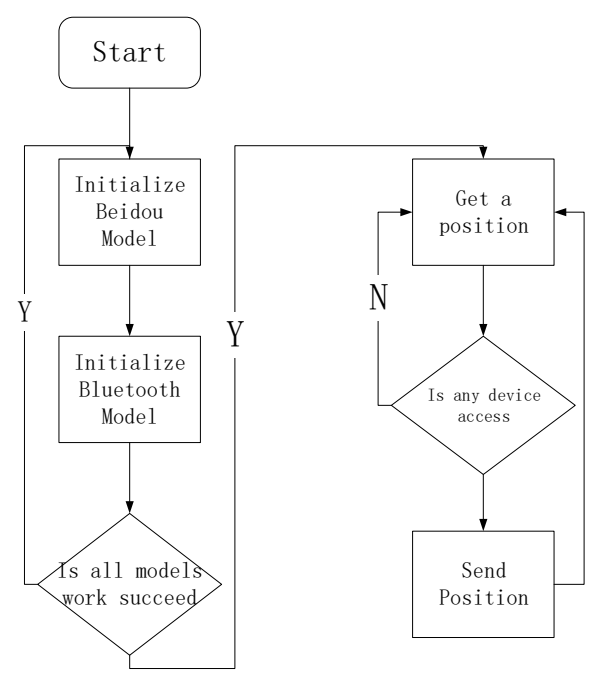

Fig. 2 Beidou terminal system design diagram 
Figure 2 illustrates the initialization process of the Beidou indicator, which provides a simple led indicator during normal operation. When you are able to communicate properly, you can view location information from your phone software.

3.2.2 Android client design: shown in figure 3

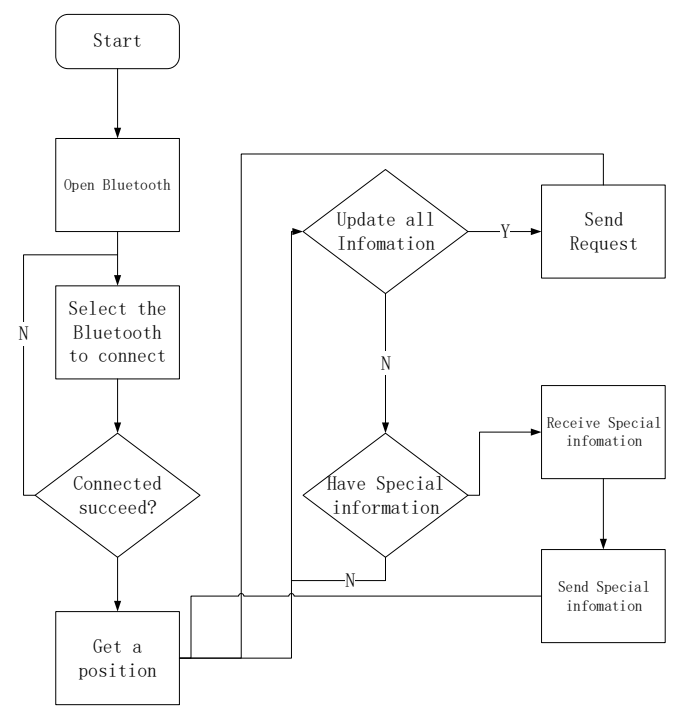

Figure 3 Android client systemdesign

Figure 3 illustrates the initialization process for the Android phone side of OSA patients. The Beidou indicator mark sends a simple condition message to the mobile terminal, and presses the button SOS on the Beidou terminal to immediately send the condition information to the fixed number already stored in the Beidou indicator. The short message content includes Beidou location information and simple disease information. The Beidou indicator uses the Beidou module to send information. OSA patients can use $4 \mathrm{G}$ mobile phones to update data and display location and SMS status information for OSA patients.

\section{Data transmission}

3.3 The server reception broadcast process is shown in figure 4

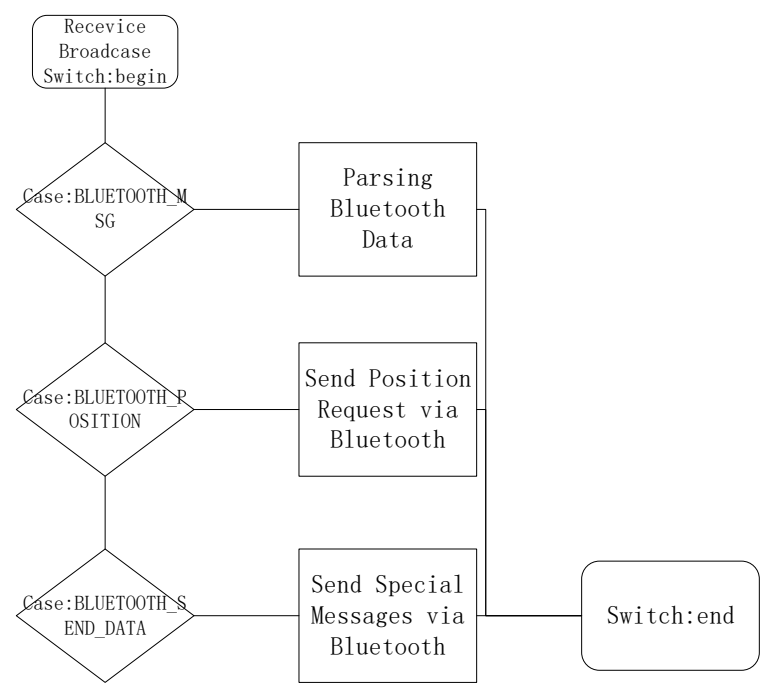

Figure 4 server receiving broadcast flow chart

The default encoding mode for Android phones is utf8 , which is used for serial communication to receive and send gdb32 encoded Chinese data. When you need to convert an object to a String object, the encoding system may produce inconsistencies, so the server also encodes and converts characters when performing transceiver control. The Server still does not stop when the Activity application is paused, so receiving information should not stop when the application is not in use at this time. Activity needs to bind to Server.

3.3.1 Design and implementation of terminal equipment

\section{3.2 functional service implementation}

Terminal function service flow, as shown in figure 5:

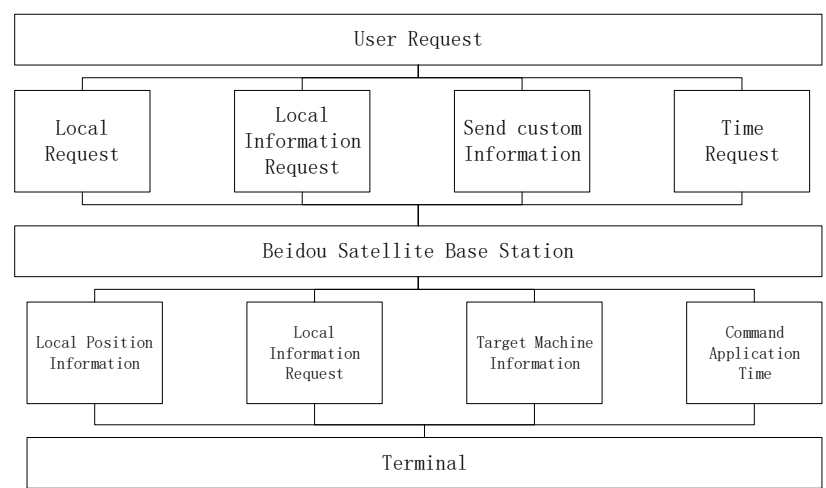

Fig. 5 Beidou terminal function service flow chart

\section{EXPERIMENTAL STUDY}

\section{1 implementation platform}

The Beidou indicator uses the STM32F103RBT chip development board. The code running on the beacon is compiled on the mdk4.9 platform and downloaded using the CooCox development tool. Android phones use android stdio as the development platform for the Android sdk version. The entire mark is shown in Figure 6.

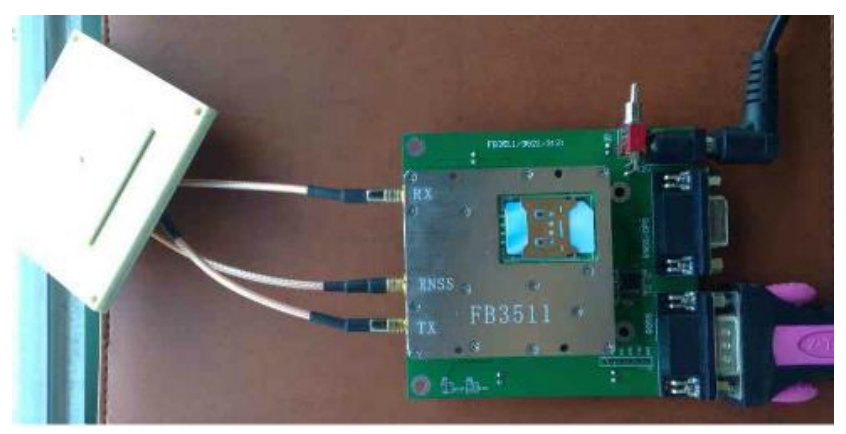

Fig. 6 Beidou interactive location-indicating machine diagram

4. 2 Android client 
The Beidou indicator uses a switch control to control the $\mathrm{s}$ witch of the Bluetooth module. If Bluetooth is turned on, you can choose to connect to the Beidou, as shown in Figure 7.

\section{Which One is Beidou's Bluetooth}

\section{BDtest}

Fig. 7 terminal selected to connect to Beidou terminal

Send success messages and location for success as shown in figure $8 \&$ figure 9

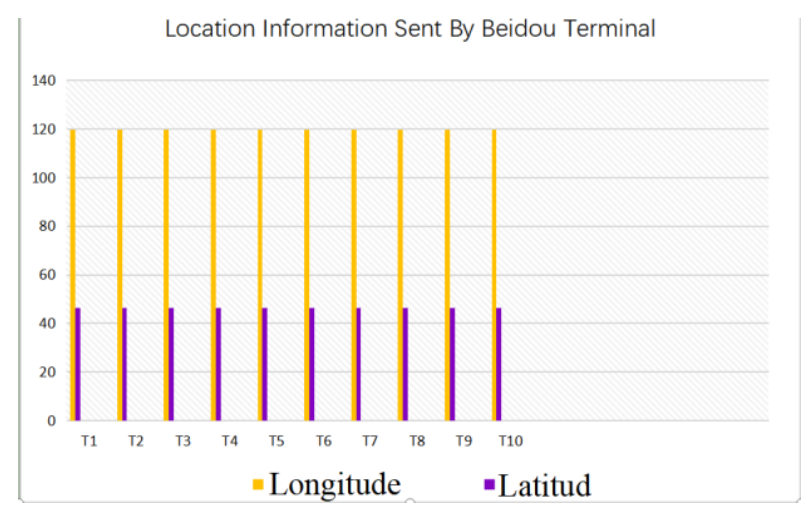

Fig. 8 success effect of Beidou sending information

\begin{tabular}{|l|l|l|l|}
\hline & Time & Longitude & Latitude \\
\hline T1 & $46: 32: 38.6$ & $119^{\circ} 46^{\prime} 46.6^{\prime \prime}$ & $46^{\circ} 46^{\prime} 37.46^{\prime \prime}$ \\
\hline T2 & $46: 34: 37.9$ & $119^{\circ} 46^{\prime} 45.9^{\prime \prime}$ & $46^{\circ} 46^{\prime} 36.97$ \\
\hline T3 & $46: 36: 38.2$ & $119^{\circ} 46^{\prime} 45.8^{\prime \prime}$ & $46^{\circ} 46^{\prime} 37.11^{\prime \prime}$ \\
\hline T4 & $46: 38: 38.8$ & $119^{\circ} 46^{\prime} 46.3^{\prime \prime}$ & $46^{\circ} 46^{\prime} 37.19^{\prime \prime}$ \\
\hline T5 & $46: 40: 38.1$ & $119^{\circ} 46^{\prime} 46.1^{\prime \prime}$ & $46^{\circ} 46^{\prime} 37.32^{\prime \prime}$ \\
\hline T6 & $46: 42: 38.3$ & $119^{\circ} 46^{\prime} 46.6^{\prime \prime}$ & $46^{\circ} 46^{\prime} 37.29^{\prime \prime}$ \\
\hline T7 & $46: 44: 38.2$ & $119^{\circ} 46^{\prime} 46.6^{\prime \prime}$ & $46^{\circ} 46^{\prime} 37.45^{\prime \prime}$ \\
\hline T8 & $46: 46: 38.3$ & $119^{\circ} 46^{\prime} 46.5^{\prime \prime}$ & $46^{\circ} 46^{\prime} 37.48^{\prime \prime}$ \\
\hline T9 & $46: 48: 38.1$ & $119^{\circ} 46^{\prime} 46.4^{\prime \prime}$ & $46^{\circ} 46^{\prime} 37.47^{\prime \prime}$ \\
\hline T10 & $46: 50: 38.5$ & $119^{\circ} 46^{\prime} 46.5^{\prime \prime}$ & $46^{\circ} 46^{\prime} 37.40^{\prime \prime}$ \\
\hline
\end{tabular}

Fig. 9 success effect of Beidou sending information

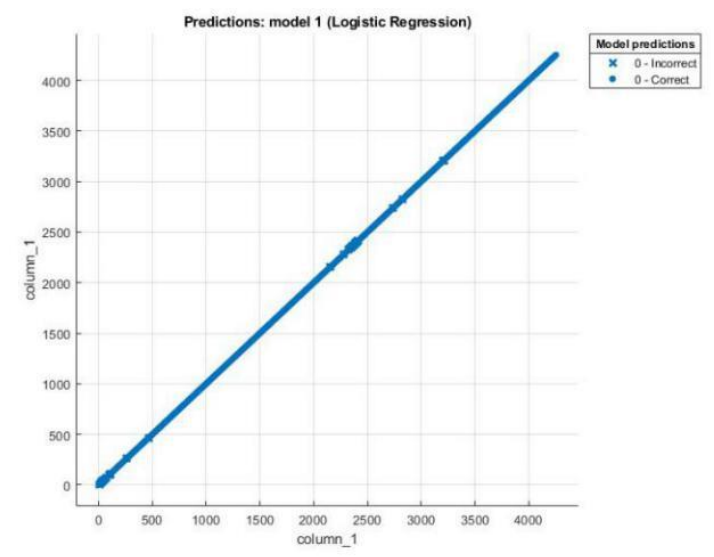

Fig 1. Scatter plot from logistic regression

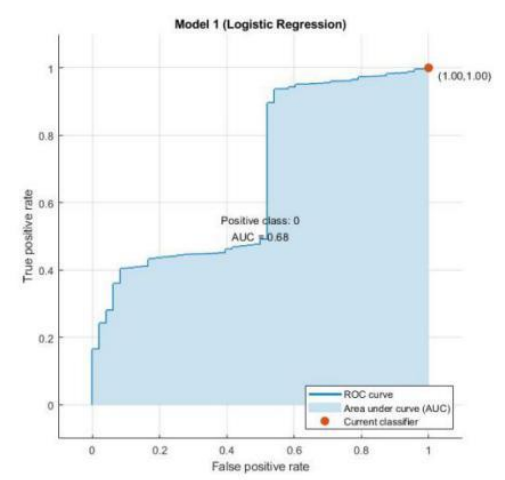

Fig 2. ROC curve from logistic regression

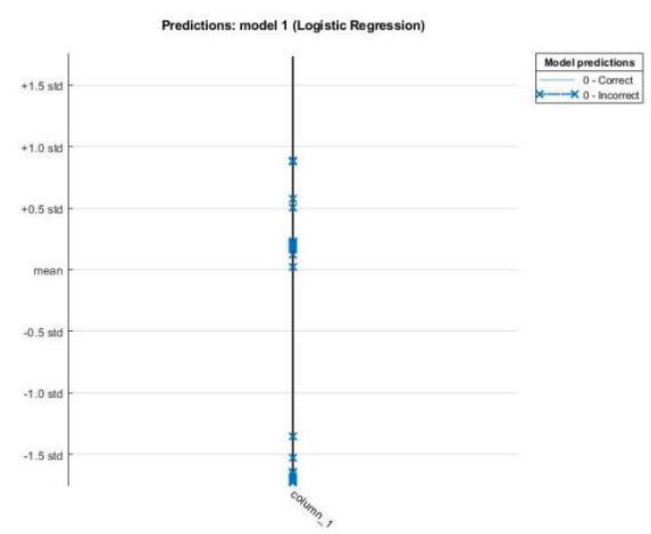

Fig 3. Parallel coordinate plot from the results of logistic regression

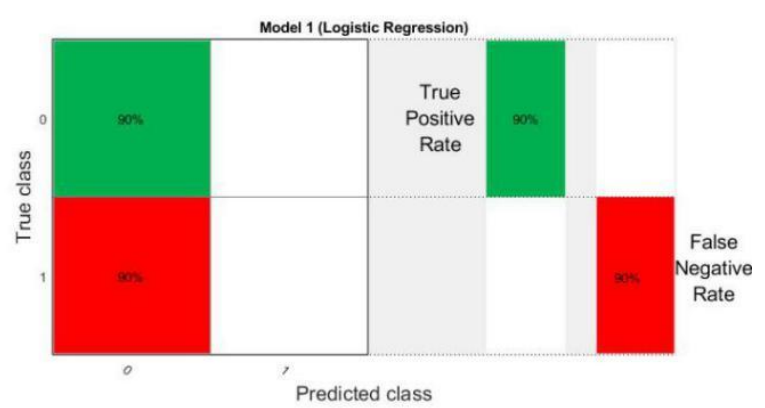

Fig 4. Confusion matrix plot from logistic regression 


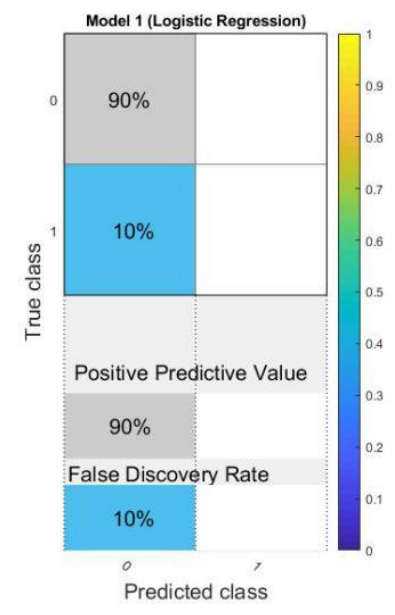

Fig 5. Confusion matrix plot with positive predictive value and false discovery rate from logistic regression

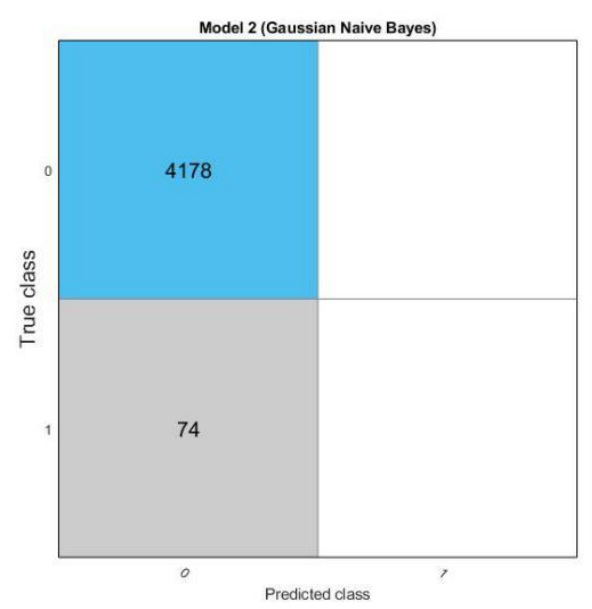

Fig 6. Confusion matrix plot with true class and predicted class from logistic regression

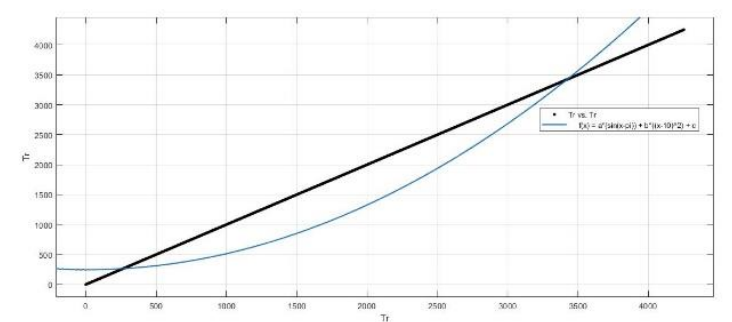

Fig 7. Customized fitting model with $\mathrm{f}(\mathrm{x})=\mathrm{a}^{*}(\sin (\mathrm{x}-\mathrm{pi}))+$ $\mathrm{b}^{*}\left((\mathrm{x}-10)^{\wedge} 2\right)+\mathrm{c}$

A customized fitting model is shown in Fig. 7. The coefficients of the model are $a=6.989, b=0.0002727$ and $\mathrm{c}=247.1 \quad(237.6,256.7)$. The R-square value: 0.9222 and root mean squared error is 382.8 .

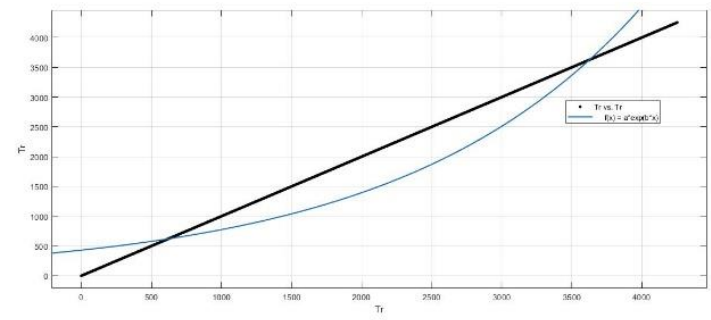

Fig 8. Exponential fitting model

The dataset is also fitted with exponential fitting $(\mathrm{f}(\mathrm{x})=$ $\mathrm{a}^{*} \exp \left(\mathrm{b}^{*} \mathrm{x}\right)$ ). The coefficients of the model are $\mathrm{a}=430.5$, and $\mathrm{b}=0.0005877$. The R-square value: 0.8975and root mean squared error is 439.5. The Gauss fitting model is shown in Fig. 9. The equation of gauss fitting model is $f(x)=$ $\mathrm{a} 1 * \exp \left(-((\mathrm{x}-\mathrm{b} 1) / \mathrm{c} 1)^{\wedge} 2\right)$. The coefficients of the model are a1 $=3855, \mathrm{~b} 1=4005$ and $\mathrm{c} 1=2310$. The R-square value: 0.9795 and root mean squared error is 196.6.

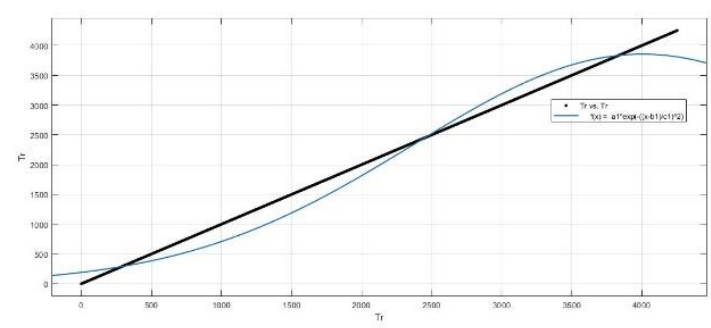

Fig 9. Gauss fitting model

The motivation of the present work was to classify patients with OSA or without OSA. The goal of the work can be divided in two categories which is binary in nature. We used logistic regression method to create classification model. The Apnea-ECG dataset have has been divided into training and testing data. Out of these, $70 \%$ data is used to train the model and $30 \%$ data was used to test the results. The simulation results confirmed that sleep disorder can be predicted and classified using logistic regression. The classification accuracy is found to be $90 \%$.

\section{V.CONCLUSIONS}

The OSA sleep disorder patient monitoring systemis based on the IoT framework structure. The entire system provides real-time disease reporting information, precise location information, and physician assistance information for OSA patients. The terminal hardware is realized by the Beidou indicator module, the $4 \mathrm{G}$ mobile communication module, and the BLE communication module. Through the secondary development of the Android mobile phone SDK development kit, it is convenient for users to operate. The special operation of shielding the Beidou indicator is made to make this OSA patient monitoring and rescue system easier to integrate into the existing hospital emergency system. The systemcurrently only provides the Android mobile phone client as the input 
and receiving end of the message, which is convenient for the user to interact with the doctor in real time through the smart phone. The OSA monitoring system has self-healing capabilities and is extremely robust, providing system reliability. This systemtest shows that the OSA patient system we developed has lower power consumption and a simpler hardware composition. In addition, our OSA patient monitoring and rescue systemhas stable and simple operation while meeting design requirements. In short, our systemis an innovative application of IoT technology in modern medicine, with a particular focus on the effective management of OSA patients. In the future, we will explore how to further improve system performance by integrating more sensors to monitor OSA patients in a more comprehensive manner.

The results from simulation of the present study show that the use of machine learning techniques like logistic regression is well suited to construct a classifier for sleep disorder patients. LR has the best performance in all aspects of prediction accuracy, precision, recall, and prediction.

These high accuracy results will encourage researchers to study sleep disorder data more rigorously and to use logistic regression for binary output in more real-world classification problems. As next phase or research, we plan to used deep learning, convolutional neural network, and transfer learning to sleep disorder classification. Moreover, it is intended to collect data from hospitals and use machine learning algorithms to evaluate and predict sleep disorder. A device with IOT can be made to monitor sleep disorder in real time.

\section{ETHICAL STATEMENT}

As per ADNI protocols, all procedures performed in studies involving human participants were in accordance with the ethical standards of the institutional and/or national research committee and with the 1964 Helsinki declaration and its later amendments or comparable ethical standards. The ADNI data collection have obtained written informed consent from the participants. More details can be found at adni.loni.usc.edu. (This article does not contain any studies with human participants performed by any of the authors).

\section{ACKNOWLEDGMENT}

This work is supported by the Fujian Provincial Scholarship Council, the National Science Foundation of China (No.61773124) the Fujian Natural Science Foundation (2018J01796), the Fujian Science and Technology Department Foundation(2019I1009), Project of Youth Foundation of Fujian Education Department (JAT191104), the Foundation of Fuzhou University Zhicheng College (KJ20190001), in part by The Open Fund of Provincial Key Laboratory of Eco-Industrial Green Technology-WuyiCollege.

\section{REFERENCES}

[1] Mencar, C., et al., Application of machine learning to predict obstructive sleep apnea syndrome severity. Health Informatics Journal, 2019: p. 1-20.

[2] Nikolaidis, K., et al., Learning from Higher-Layer Feature Visualizations. p. arXiv:1903.02313v1.2019.

[3] Ravichandran, V., et al., RespNet: Adeep learning model for extraction of respiration from photoplethysmogram. p. arXiv:1902.04236v2 [eess.SP] 20 Feb 2019.

[4] Van Steenkiste, T., et al., Automated Sleep Apnea Detection in Raw Respiratory Signals using Long Short-Term Memory Neural Networks. IEEE Journal of Biomedical and Health Informatics, 2018: p. 1-11.

[5] Cen, L., et al., Aut omatic System for Obstructive Sleep Apnea Events Detection Using Convolutional Neural Network. Conference proceedings : . Annual International Conference of the IEEE Engineering in Medicine and Biology Society. IEEE Engineering in Medicine and Biology Society. Annual Conference, 2018. 2018: p. 3975 .

[6] Islam, S.M., et al. Deep Learning of Facial Depth Maps for Obstructive Sleep Apnea Prediction. in 2018 International Conference on Machine Learning and Data Engineering (iCMLDE). 2018: IEEE.P 154-157.

[7] Falco, I.D., et al. Deep Neural Network Hyper-Parameter Setting for Classification of Obstructive Sleep Apnea Episodes. in 2018 IEEE Symposium on Computers and Communications (ISCC). 2018: IEEE.P 01187-01192.

[8] Lakhan, P., et al., Deep Neural Networks with Weighted Averaged Overnight Airflow Features for Sleep Apnea-Hypopnea Severity Classification. 2018: p. arXiv:1808.10845v1 [eess.SP]31 Aug 2018.

[9] Stephen McCloskey B, R.H.B., K.B. Irena and A.B. Jeffries, Detecting Hypopnea and Obstructive Apnea Events Using Convolutional Neural Networks on Wavelet Spectrograms of Nasal Airflow. D. Phung et al. (Eds.): PAKDD 2018, LNAI 10937, pp. 361 - 372, 2018., 2018.

[10] Jansen, C., et al., Feature relevance in physiological networks for classification of obstructive sleep apnea. Physiol Meas, 2018. 39(12): p. 124003.

[11] Urtnasan, E., J.U. Park and K.J. Lee, Multiclass classification of obstructive sleep apnea/hypopnea based on a convolutional neural network from a single-lead electrocardiogram. Physiol Meas, 2018. 39(6): p. 065003.

[12] Hinton, G., Deng, L., Yu, D., Dahl, G. E., Mohamed, A. R., Jaitly,N., and Kingsbury, B., Deep neural networks for acoustic modeling in speech recognition: The shared views of four research groups.IEEE Signal Process. Mag. 29(6):82 - 97, 2012..

[13] LeCun, Y., Bengio, Y., and Hinton, G., Deep learning. Nature 521(7553):436 - 444, 2015.https://doi.org/10.1038/nature14539.

[14] Pathinarupothi, R.K., et al. Instantaneous heart rate as a robust feature for sleep apnea severity detection using deep learning. in 2017 IEEE EMBS International Conference on Biomedical \& Health Informatics (BHI). 2017: IEEE 293-296.

[15] Pathinarupothi, R.K., et al. Single Sensor T echniques for Sleep Apnea Diagnosis Using Deep Learning. in 2017 IEEE International Conference on Healthcare Informatics. 2017: IEEE. 524-529.

[16] L. C. Siqueira, A. F. L. Jacob, and Á. L. de Santana, "Multimodal LowInvasive System for Sleep Quality Monitoring and Improvement BT Beyond the Internet of Things: Everything Interconnected," J. M. Batalla, G. Mastorakis, C. X. Mavromoustakis, and E. Pallis, Eds. Cham: Springer International Publishing, 2017, pp. 223-242. 
[17] Y. Nam, Y. Kim, and J. Lee, "Sleep Monitoring Based on a TriAxial Accelerometer and a Pressure Sensor," Sensors (Basel)., vol. 16, no. 5, p. 750, May 2016.

[18] Zhang Li Hua. The Chinese Beidou is the world's third-proven satellite navigation system [N]. People's Daily Overseas Edition, 2014 -03-03.

[19] Jiang even move, Mr Bao Feng. The Beidou" into a global satellite navigation systems, one of the four core .[N]. Jiefangjun Bao -11-23. 2015 .

[20] Cloud into the Beidou system public service performance specification (version 1.0), summarized the [J]. satellite applications, 2014, (4): 4245 .

[21] Mr Ho. Wireless below-standard meter design [D]. Harbin Engineering University, $2015: 23-28$

[22] Liu Bang sound. GMDSS communications business [M].: Dalian, Dalian Maritime University Press, 15 :68-78 20

[23] $\mathrm{Mr}$ de light. On the basis of the Beidou vehicle transport monitoring scheduling system for the design and implementation of the Beijing Chemical Industry University [D].20 14:30-35.

[24] Li Xiao light. China's satellite industry depth studies [R]. Shanghai Shenyin Wanguo Securities Research Institute Co., 201 5 :33-39.

[25] Wang Li-chan. The Beidou satellite navigation system - Current Status and development trend of $[\mathrm{J}]$. study the town building theory research (electronic), 2014, (8) : 66-71

[26] The Beidou satellite navigation system to provide regional services today formally [N]. China News 2015 -12-27.

[27] Mobutu Yu. STM32 self-study notes [M]. Beihang University Press, $2015: 88-108$.

[28] Matt richardson/shawn wallace. fall in love with a raspberry PI [M]. Lee where the Greek translation of science books .2015 : 120-150.

[29] St Stmicroelectronics .STM32F10X Firmware Library Chinese explanation of V2.0[eb/ol].um0427.201 User Manual 5: 200-235

[30] The AT command reference [EB/OL] .microsoft Technical Support .2014:88-95.

[31] The android overview [EB/OL] open handset alliance.20 15: 180-205.

[32] Mr Ding, Zhang Qin, a small red, satellite navigation and positioning theory [M] , Wuhan, Wuhan University Press, 2015:36-56.

[33] B. Ma, C. Li, Z. Wu, Y. Huang, A. van der Zijp-Tan, S. Tan, D. Li, A. Fong, C. Basetty, G.M. Borchert, and J. Huang*, "A PWM-based muscle fatigue detection and recovery system," Proc. 2018 IEEE International Conference on Bioinformatics and Biomedicine (BIBM18), pp. 1013-1016, Madrid, Spain, Nov. 2018.

\section{AUTHOR BIOGRAPHY}

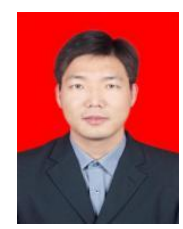

Liangming Cai received the M.S. degree in Electronic and Communication Engineering, Huazhong University of Science and Technology, Wuhan, China. Now he is an associate professor at Fuzhou University Zhicheng College and is currently pursuing the $\mathrm{PhD}$ degree with the College of Physics and Information Engineering from Fuzhou University,Fuzhou, China. He was a Visit scholar in School of Computing in University of South Alabama from September 2018 to August 2019 and in Software School of Xiamen
University from September 2015 to August 2016. His research interests include IOT(Internet of Thing), $\mathrm{AI}$ (Artificial Intelligence) and Big Data,Time-series Modeling Embedded System,Biomedical Signal Analysis, OSA(Obstructive Sleep Apnea).

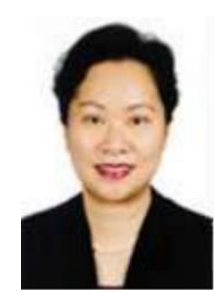

Min Du was born in Fujian, China. She received the PhD. degree in electrical engineering from Fuzhou University, Fuzhou, China, in 2005, where she has been the Associate Director of the Fujian Key Laboratory of Medical Instrumentation and Pharmaceutical Technology, since 2007, and is currently a Professor and a Doctoral Supervisor.Her research interests include biomedical signal detection and analysis, smart instruments, and photo electrical systems. 